\title{
External continuous ECG versus loop recording for atrial fibrillation detection in patients who had a stroke
}

\author{
Michala Herskind Sejr, ${ }_{1}^{\oplus 1}$ Ole May, ${ }^{1}$ Dorte Damgaard, ${ }^{2}$ Birgitte Forsom Sandal, ${ }^{3}$ \\ Jens Cosedis Nielsen ${ }^{4}$
}

- Additional material is published online only. To view please visit the journal online (http://dx.doi.org/10.1136/ heartjnl-2018-314186).

'Department of Cardiology, Cardiovasculair Research Unit, Regional Hospital West Jutland, Herning, Denmark ${ }^{2}$ Department of Neurology, Aarhus Universitetshospital, Aarhus, Denmark

${ }^{3}$ Department of Neurology, Regional Hospital West Jutland, Denmark

${ }^{4}$ Department of Cardiology, Aarhus University Hospital, Aarhus, Denmark

Correspondence to Michala Herskind Sejr, Department of Cardiology, Cardiovasculair Research Unit, Herning 7400, Denmark; michhers@rm.dk

Received 24 September 2018 Revised 13 January 2019 Accepted 17 January 2019 Published Online First 21 March 2019

\begin{abstract}
Background Detection of atrial fibrillation (AF) in patients who had ischaemic stroke and transient ischaemic attack (IS/TIA) is recommended. We aimed to compare external loop recording (ELR) against simultaneous continuous ECG recording for AF detection in patients who had acute IS/TIA and determine sensitivity, specificity and positive predictive value of $A F$ detection using ELR. We hypothesised ELR to detect 15\% fewer patients with AF than continuous ECG recording. Methods In this prospective cohort study, we included 1412 patients who had acute IS/TIA without prior AF. Monitoring was 48 hours. Primary outcome was AF $>30$ s. Cardiologist verified AF in continuous ECG was gold standard.
\end{abstract}

Results In continuous ECG, 38 (2.7\%) patients had AF. ELR automatically categorised $219 / 1412$ patients (15.5\%) with AF, including 32/38 (85\%) patients with AF in continuous ECG. After cardiologist adjudication of ELR recordings, AF was diagnosed in 57/219 patients, of which $32(56 \%)$ had AF in continuous ECG. For adjudicated AF detection by ELR, sensitivity was $84 \%$, $95 \% \mathrm{Cl}(69 \%$ to $94 \%)$, specificity was $98 \%, 95 \% \mathrm{Cl}$ (97\% to $99 \%$ ) and positive predictive value was $56 \%$, $95 \% \mathrm{Cl}(42 \%$ to $69 \%)$.

Conclusion Automatic AF detection with ELR results in an AF diagnosis in more than five patients without $A F$ for each patient with AF as verified in continuous ECG. For adjudicated AF detection by ELR, sensitivity was confirmed to $84 \%$ and specificity $98 \%$. Automatic ELR as investigated in this study may be considered to rule out AF, but it is not suitable as a single monitoring device for AF screening in patients early after stroke.

Trial registration number NCT02155907.

\section{INTRODUCTION}

Stroke is the second leading cause of death and disability worldwide with 15 million new cases each year. ${ }^{1}$ It is estimated that $15 \%$ of all strokes are caused by atrial fibrillation (AF). ${ }^{2}$ With a growing elderly population, prevalence of AF increases, and estimated $9 \%$ of people older than 65 years have $\mathrm{AF}^{3} \mathrm{AF}$ entails up to five times higher risk of ischaemic stroke and increased risk of recurrent stroke. ${ }^{4}$ Strokes related to AF cause higher mortality, worse functional outcome and longer hospitalisations. ${ }^{5}$

It is well documented that oral anticoagulation (OAC) treatment reduces stroke risk significantly in patients with $\mathrm{AF}^{6}{ }^{6}$ and detection of $\mathrm{AF}$ in patients who had a stroke is of utmost importance. The paroxysmal and often asymptomatic character of AF is a huge challenge in that setting. Recent guidelines recommend monitoring of 3-30 days in patients who had a stroke to search for $\mathrm{AF}^{78}$ There is, however, no consensus about best method to detect non-persistent AF.

Continuous ECG recording is widely accepted as gold standard in ambulatory heart rhythm analysis. Recently, a considerable range of heart rhythm detection devices have been marketed. External loop recorders (ELRs) hold several practical and economic advantages compared with continuous ECG recording, offering up to 32 days continuous monitoring, high patient comfort and automatic AF-detecting software. Such devices in general are not validated against simultaneous continuous ECG recording in relevant patient cohorts. We aimed to compare 48 hours ELR monitoring against simultaneous continuous ECG recording for AF detection in a large cohort of patients who had a stroke and determine sensitivity, specificity and positive predictive value of AF detection using ELR.

\section{METHODS}

Prospectively, consecutive patients were recruited from Department of Neurology, Regional Hospital West Jutland in Denmark during June 2013 to December 2017. Inclusion criteria were acute ischaemic stroke or transient ischaemic attack (TIA) with first symptoms within 1 week, age $\geq 60$ years, no AF on 12-lead admission ECG, no prior AF according to International Classification of Diseases codes (ICD-10) from outpatient clinic visits, hospitalisations or review of medical records, no active cancer, no implanted pacemaker, no expected low compliance or precedent participation in this study and written informed consent. Neurologists ascertained diagnosis of stroke or TIA and cerebral imaging (CT or MRI) was obtained for all patients. TIA was defined as stroke-like symptoms lasting shorter than 24 hours. AF was defined according to current guidelines, as an atrial arrhythmia with irregular intervals between $\mathrm{R}$ waves, without detectable normal P waves and lasting more than 30 s. $^{9}$

We started simultaneous continuous ECG recording and ELR monitoring to obtain continuous recording for 48 hours. An illustrating picture is presented in supplementalry appendix 2 . 
Table 1 Ischaemic stroke: includes the ICD-10 diagnosis codes: DI639, DI649, DI632, DI453 and DI634

\begin{tabular}{lc}
\hline Baseline characteristics ( $\mathrm{n}=1412)$ & \\
\hline Male, $\mathrm{n}(\%)$ & $797(56.0)$ \\
\hline Age, years, mean (SD) & $562(3.79 .8)$ \\
\hline $\mathrm{TIA}, \mathrm{n}(\%)$ & $849(60.2)$ \\
\hline Ischaemic stroke, $\mathrm{n}(\%)$ & $825(58.4)$ \\
\hline Hypertension, $\mathrm{n}(\%)$ & $20(1.4)$ \\
\hline Heart failure (ejection fraction $<40 \%), \mathrm{n}(\%)$ & $202(14.3)$ \\
\hline Diabetes, $\mathrm{n}(\%)$ & $67(4.8)$ \\
\hline Claudication, $\mathrm{n}(\%)$ & $345(24.6)$ \\
\hline Current smoker, $\mathrm{n}(\%)$ & $150(21.3)$ \\
\hline Systolic blood pressure, mm Hg, mean (SD) & $81(12.2)$ \\
\hline Diastolic blood pressure, mm Hg, mean (SD) & $26.1(4.5)$ \\
\hline BMI, $\mathrm{kg} / \mathrm{m}^{2}$, mean (SD) & $40.8(8.8)$ \\
\hline HgbA1c, mmol/mol, mean (SD) & $5.12(1.26)$ \\
\hline Total cholesterol, mmol/L, mean (SD) & $493(34.9)$ \\
\hline Statins, $\mathrm{n}(\%)$ & $361(26.6)$ \\
\hline Aspirin, $\mathrm{n}(\%)$ & $32(2.3)$ \\
\hline Dipyridamole, $\mathrm{n}(\%)$ & $11(0.78)$ \\
\hline Anticoagulation therapy, $\mathrm{n}(\%)$ & $125(8.9)$ \\
\hline Clopidogrel, $\mathrm{n}(\%)$ & \\
\hline
\end{tabular}

Reported medication was before stroke.

BMI, body mass index; HgA1c, hemoglobin A1 c; TIA, transient ischaemic attack with symptoms $<24$ hours.

We used R.Test Evolution 4 (NorDiaTech, Paris, France) as ELR. This device is non-invasive and records heart rhythm using two skin electrodes attached over sternum and cardiac apex. ELR recorders were attached by nurses after manufacturer's recommendations. The ELR analyses segments of 64 consecutive RR intervals (intervals between $\mathrm{R}$ waves), when at least two-thirds of these intervals are irregular, categorises heart rhythm as $\mathrm{AF}$ and stores a recording of AF episode in memory. Depending on heart rate, the ELR is able to categorise AF episodes lasting from approximately $25 \mathrm{~s}$, thereby suitable for detecting AF exceeding $30 \mathrm{~s}$. Storing capacity is $60 \mathrm{~min}$, and if this is exceeded, only the most characteristic AF episodes are kept. AF episodes with fastest heart rates are kept in memory. We adjusted ELR according to manufacturer's recommendations. We saved 1 min recording per $\mathrm{AF}$ episode, allowing for a maximum of $54 \mathrm{AF}$ recordings per patient, while $6 \mathrm{~min}$ were spared for storage of episodes of other arrhythmia.

Three experienced members of the research team (MHS, $\mathrm{OM}$ and $\mathrm{JCN}$ ) each reviewed and classified as $\mathrm{AF}$ or non-AF all recordings automatically classified as AF by the ELR. In case of ambiguity, agreement was reached by consensus. Analysis of ELR findings was blinded for continuous ECG recording results. The continuous ECG recorder used was Life Card CF digital ECG recorder from Spacelabs Healthcare Diagnostic Cardiology (Washington, USA). Nurses trained and experienced in analysing continuous ECG recordings reviewed recordings. Episodes classified as AF were verified by the three members of the research team. Analysis was blinded to ELR results.

\section{Ethics}

The study complies with Declaration of Helsinki and was reported to ClinicalTrials.gov.

\section{Statistical analysis}

Baseline and follow-up data were presented as numbers (percentages) and means (SD) if normally distributed; otherwise, data were presented as median (IQR). We used STATA V.15 from StataCorp LP for statistical analyses.

Continuous ECG recording was defined as gold standard as we aimed to estimate sensitivity, specificity and positive and negative predictive values of AF detection using ELR, both for automatically detected $\mathrm{AF}$ and after review of AF episodes by experienced cardiologists. Detection of AF was categorised as binary outcome.

We used the DIAGT Stata V.15 module to report sensitivity, specificity, positive predictive value and negative predictive value from our contingency tables (table 2), all with exact binomial confidence intervals. Here we compared AF in continuous ECG to AF registered in ELR recordings, as well as AF in continuous ECG to validated AF in ELR. AF in continuous ECG was set as gold standard in the STATA command.

We expected 48 hours continuous ECG recording to detect AF in $10 \%$ and ELR in $8.5 \%$ of patients, thus expecting a sensitivity of $85 \% .^{10}$

Hypothesis: 2-day ELR has a sensitivity of $85 \%$ compared with 2-day continuous ECG recording (gold standard) to detect $\mathrm{AF}$ in a consecutive population with recent stroke/TIA.

Null hypothesis: 2-day ELR sensitivity differs from $85 \%$ compared with 2 day continuous ECG recording (gold standard) in a consecutive population with recent stroke/TIA.

\section{Sample size}

We estimated a required sample size of 1480 patients in order to detect an ELR sensitivity difference of at least $10 \%$ from a hypothesised sensitivity of 0.85 , assuming detection of $\mathrm{AF}$ in $10 \%$ of the population. A binomial (two sided) test comparing one proportion (the sensitivity of 0.85 ) to a reference value $(0.75)$ was used. A priori, sample size was not calculated for specificity comparisons.

\section{RESULTS}

Flow diagram depicting numbers of patients who had ischaemic stroke and TIA older than 60 years hospitalised in our department during the inclusion period is shown in figure 1. We included 1507 patients consecutively. We excluded 95 patients, in whom ELR recording was not started correctly, resulting in a study cohort of 1412 patients with simultaneous 48 hours ELR and continuous ECG recording. Baseline characteristics of study cohort is presented in table 1. Eight hundred and forty-nine patients (60.2\%) had ischaemic stroke and 562 patients (39.8\%) had TIA. Median (IQR) ELR monitoring time was 48.2 (1.55) hours with duration of analysable recording of 45.1 (14.3) hours. Duration of analysable continuous ECG recordings was 48.0 (1.08) hours.

The ELR automatically categorised 219 of the 1412 patients (15.5\%) with AF. The 219 ELR recordings were reviewed by trained cardiologists among the authors and 57 (26.0\%) were

Table 2 Sensitivity, specificity, positive and negative predictive values of automatically detected and cardiologist verified AF detection by ELR with $95 \% \mathrm{Cl}$ compared with findings with 48 hours continuous ECG recording as gold standard

\begin{tabular}{lll}
\hline AF automatically detected by ELR (\%) & $\begin{array}{l}\text { AF detected by ELR and } \\
\text { cardiologist verified (\%) }\end{array}$ \\
\hline Sensitivity & $92(79$ to 98$)$ & $84(69$ to 94$)$ \\
Specificity & $87(85$ to 88$)$ & $98(97$ to 99$)$ \\
Positive predictive value & $16(11$ to 22$)$ & $56(42$ to 42$)$ \\
Negative predictive value & $99.7(99.3$ to 99.9$)$ & $99.6(99.0$ to 99.8$)$ \\
\hline
\end{tabular}




\section{Figure 1 Flow diagram of patient enrolment into study}

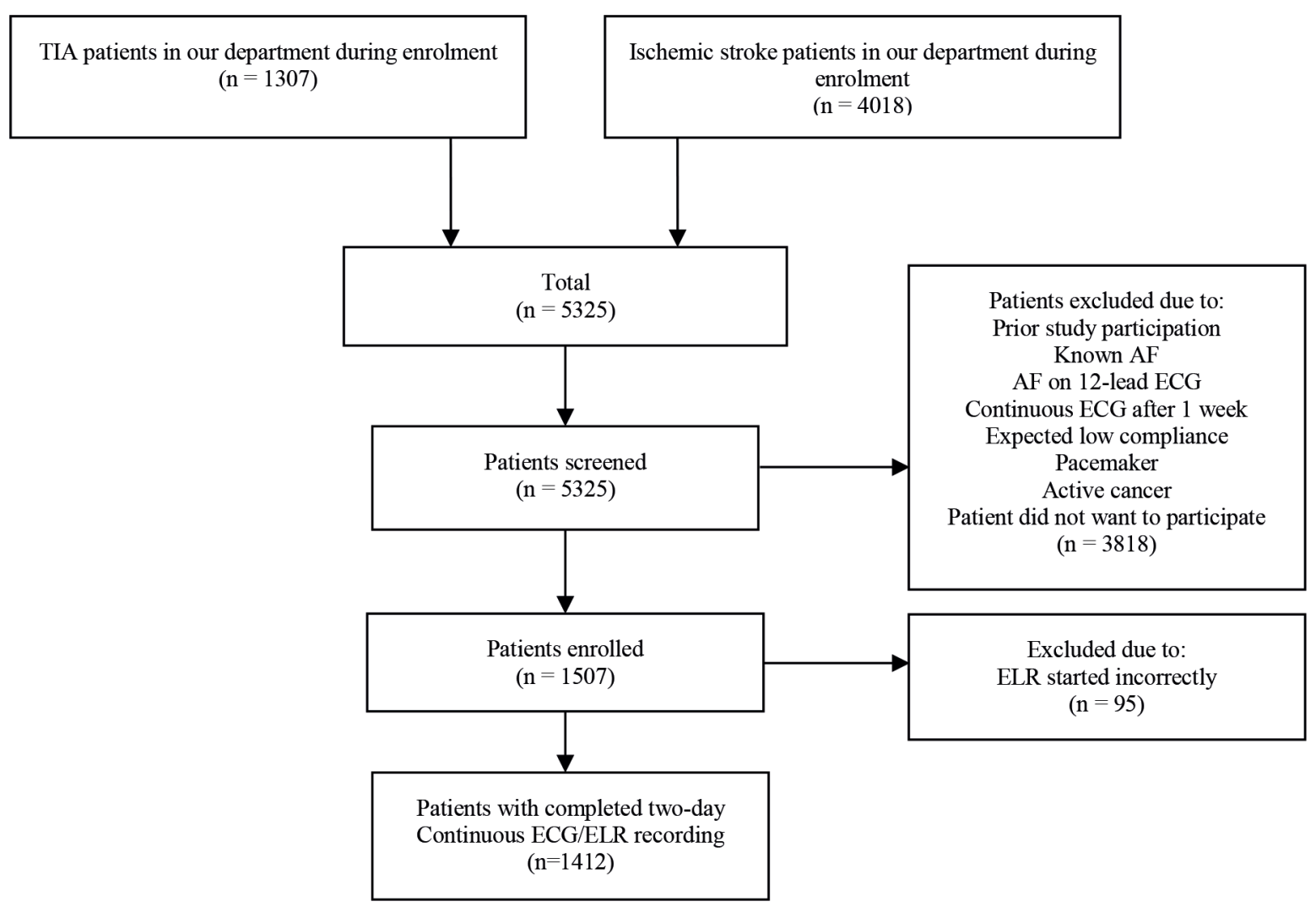

Figure 1 Flow diagram depicting numbers of patients older than 60 years hospitalised with ischaemic stroke or TIA during inclusion period. ELR, external loop recorder; TIA, transient ischaemic attack.

verified as AF, while remaining $162(74.0 \%)$ were classified as non-AF. AF detected by ELR and verified by cardiologists was prevalent in $4.0 \%(57 / 1412)$ of patients. In continuous ECG recording, we detected $\mathrm{AF}$ in 38 patients, corresponding to a prevalence of 2.7\% (38/1412). Automatically, ELR detected $12.8 \%$ (95\% CI $11.0 \%$ to $14.7 \%$ ) more patients with AF than did continuous ECG recording. After cardiologist's adjudication of AF episodes from ELR, AF prevalence was 4.0\% (57/1412), and ELR detected 1.3\% (95\% CI $0.50 \%$ to $2.1 \%$ ) more patients with AF than continuous ECG recording.

Discrepancies between the two monitoring methods' findings are illustrated in figure 2 . Of the 38 patients with AF in 48 hours continuous ECG recording, 32 were detected and verified as AF on ELR. The remaining six had either no AF detection in ELR (three patients) or had AF events recorded in ELR that were disproved by the authors and did not match time of AF on continuous ECG recording (three patients). AF durations on continuous ECG for those six patients were: 54, 80, 123, 218, 23476 and 64140 s, respectively. Sensitivity and specificity of AF detected by ELR automatically and as verified by cardiologists are presented in table 2 .

For the 25 patients recognised discordantly with non-AF in continuous ECG and AF in ELR, rhythm in ELR recording did fulfil AF criteria of irregular heart rhythm with no detectable $P$ waves and duration longer than $30 \mathrm{~s}$. We reviewed simultaneous two-channel continuous ECG recordings during the relevant periods with AF in ELR. For all 25 cases, we were able to identify $\mathrm{P}$ waves during the entire continuous ECG recording, confirming sinus rhythm with premature atrial complexes.
These 25/57 (43.9\%) ELR recordings therefore turned out falsely positive. Figure 3 presents some simultaneous ELR and continuous ECG recordings with discordant results; remaining simultaneous recordings are shown in online supplementary appendix 1. Figure 4presents two patients with AF in continuous ECG recording not detected by ELR. Considering automatic AF detections by ELR, these were truly positive in only $14.6 \%(32 / 219)$ of patients and thereby false positive in $85.4 \%$ of patients (table 2). Mean AF duration in ELR was shorter in the 25 patients where AF could not be confirmed in continuous ECG recording ( 1 hour $1 \mathrm{~min} 7 \mathrm{~s}$ ) than in the 32 patients with confirmed AF (11 hours $56 \mathrm{~min} 17 \mathrm{~s}$ ) (online supplementary appendix).

\section{DISCUSSION}

This study shows that results obtained by ELR monitoring differed markedly from results obtained by the gold standard method of continuous ECG recording while screening for AF in patients who had an acute stroke. Automatic ELR detection of $\mathrm{AF}$ results in an AF diagnosis in more than five patients without AF for each patient with continuous ECG recording-verified AF. For adjudicated AF detection by ELR, sensitivity was confirmed to $84 \%$ and specificity $98 \%$. Automatic ELR as investigated in this study may be considered to rule out AF, but it is not suitable as a single monitoring device for AF screening in patients early after stroke.

Our population is representative for patients with stroke or TIA admitted to hospital and represents a population where 


\section{Flow diagram of AF diagnosis}

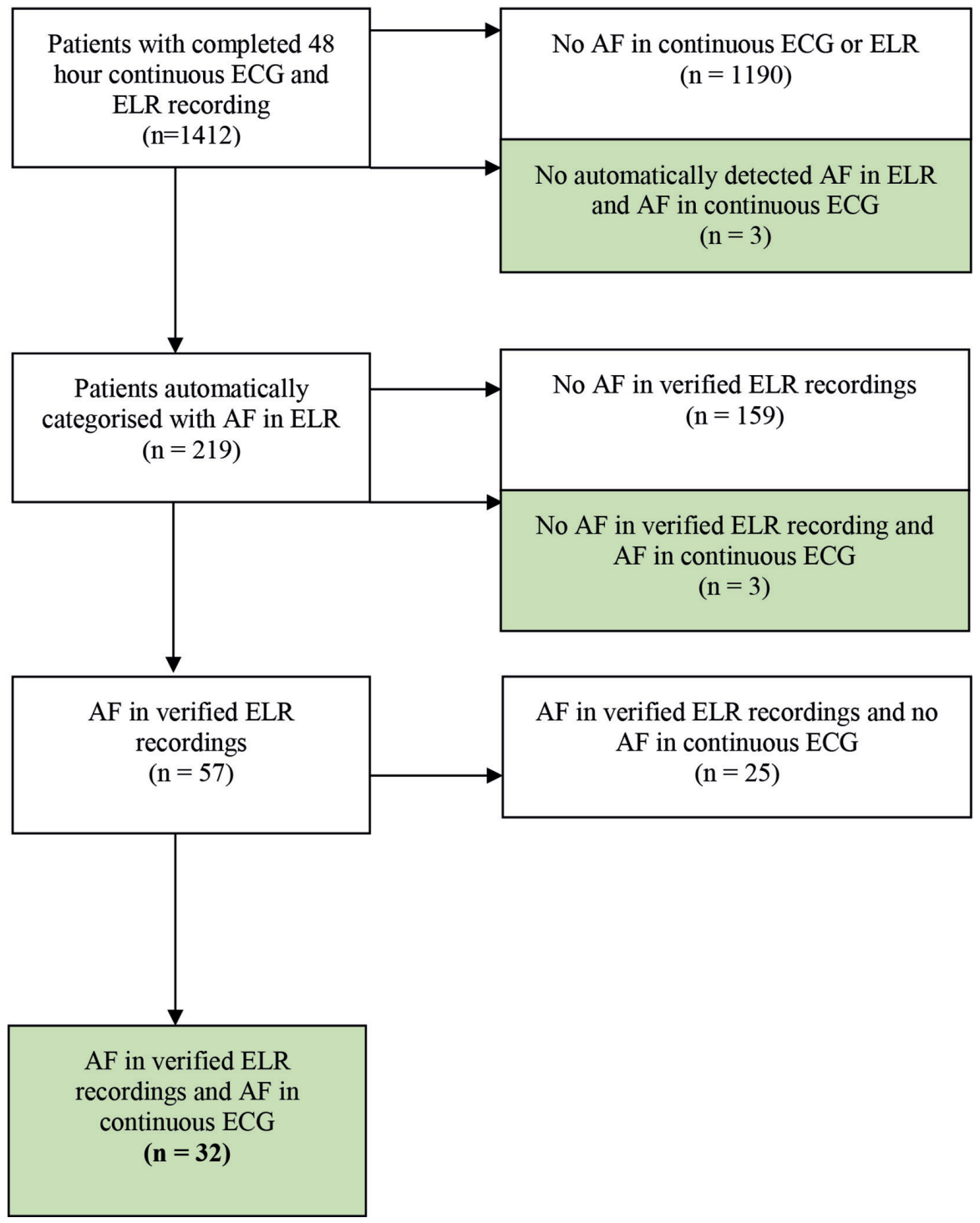

Figure 2 Flow diagram illustrating results of AF diagnostics in continuous ECG and ELR. Green marked boxes represent patients with AF in continuous ECG. AF, atrial fibrillation; ELR, external loop recorder.

screening for AF is recommended. ${ }^{7}$ The observed AF prevalence of $2.7 \%$ in 48 -hour continuous ECG recording is consistent with AF diagnosed in 1\%-5\% using 24-48 hour continuous ECG recording of stroke patients reported in a recent review. ${ }^{11}$ The high rate of false-positive detections of AF with ELR is accordant with findings in three small studies comparing ELR with simultaneous ECG recording. ${ }^{12-14}$ This finding indicates that in patients who had an acute stroke, clinicians should not rely on a diagnosis of AF established only by ELR monitoring. Not even after cardiologist verification of these recordings, as only $56 \%$ of such findings truly reflect AF.

We found that ELR detected $85 \%$ of the patients who had true $\mathrm{AF}$ as indicated by continuous ECG monitoring. The negative predictive value of ELR was high, and therefore, this monitoring device may be considered as a first-line instrument in screening patients who had an acute stroke for AF. However, in case AF is indicated by ELR, this diagnosis should be confirmed by continuous ECG monitoring or another more reliable AF detection method before clinical consequences as initiation of OAC treatment are taken.

The main reason for false-positive AF detections in the present study was automatic ELR rhythm interpretation relying on RR intervals. We included elderly patients who had a stroke/TIA, and both ageing and prior stroke are associated with increased prevalence of premature atrial and ventricular contractions. ${ }^{15}$ Premature beats are known to be even more frequent very early after stroke, ${ }^{16}$ and it cannot be ruled out that later monitoring would yield different results. One reason that continuous ECG recording is more reliable than loop recorders may be heart rhythm evaluation during the entire recording period, allowing for diagnostic identification of gradual or abrupt changes in atrial and ventricular rates.

OAC therapy is documented highly beneficial in stroke patients with AF reducing risk of recurrence. ${ }^{6}{ }^{17}$ However, anticoagulation therapy comes with an enhanced risk of bleeding and intracranial bleeding, especially in patients with earlier stroke or 

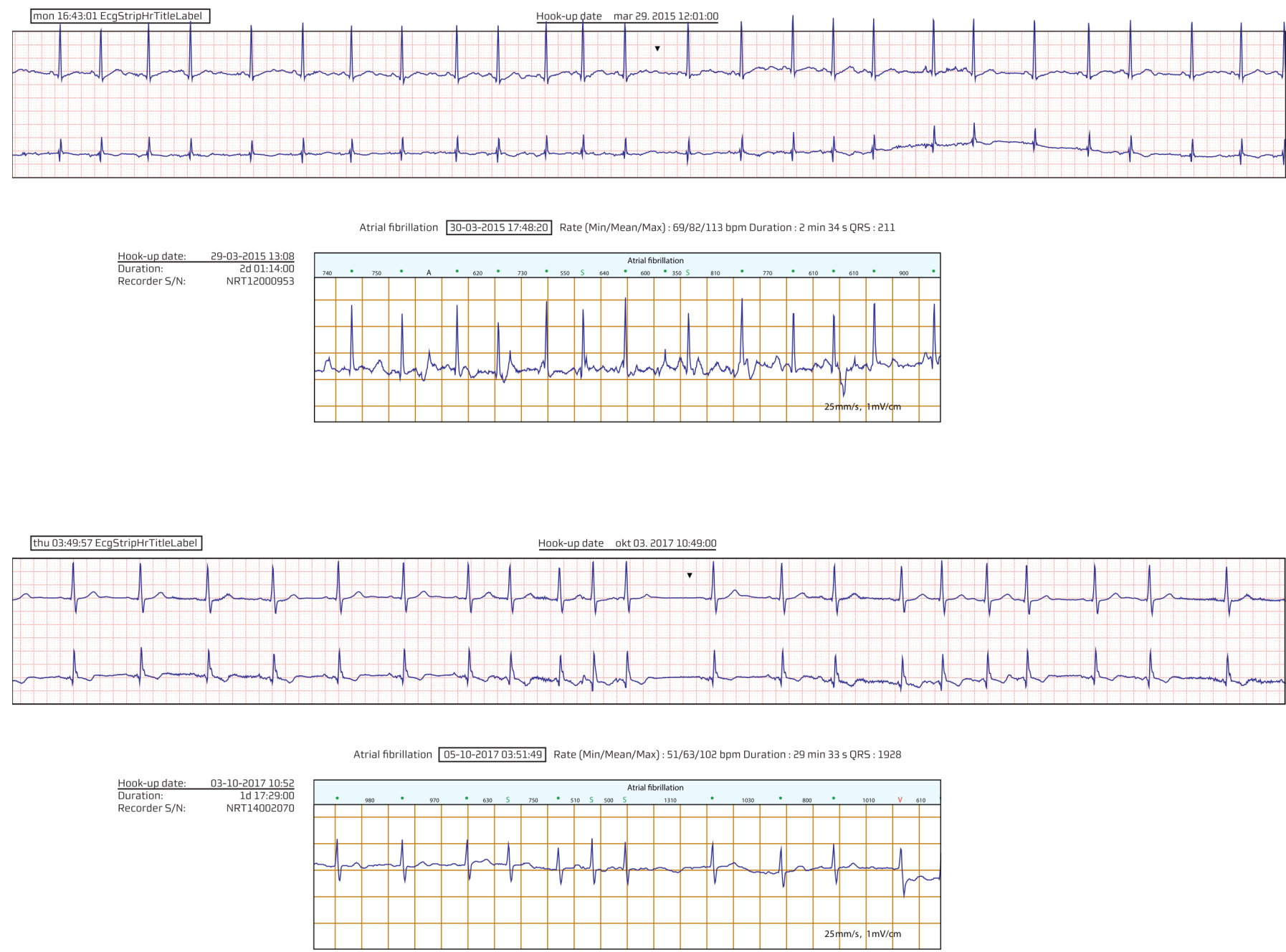

Figure 3 Examples of simultaneous 48-hour continuous ECG (top) and ELR (bottom) recordings from patients adjudicated as AF by experienced cardiologists among the authors but confirmed as sinus rhythm with supraventricular extra systoles in simultaneous 48-hour continuous ECG recording. Recorded time of AF and hook-up time are marked for all tracings. The 48-hour continuous ECG recording clock was set manually, while ELR runs at exact computer time from encoding. Some recordings have approximately 1 -hour time difference since the manual 48 -hour continuous ECG recording setting was not corrected to winter time. AF, atrial fibrillation; ELR, external loop recorder.

TIA. ${ }^{18}$ Using less reliable methods for AF detection may lead to $\mathrm{OAC}$ in patients without $\mathrm{AF}$. These patients are exposed to risks with OAC, but likely no benefit of reduced stroke risk, as supported by the recent Navigate ESUS study results. ${ }^{19}$

There are no generally accepted international guidelines on optimal duration and method of rhythm monitoring for AF detection in patients who had a stroke. We started patient inclusion in 2013. Since 2008, the European Stroke Organization recommended 24-hours continuous ECG monitoring in acute ischaemic stroke patients and additionally 24-hour continuous ECG recording for selected patients. ${ }^{20}$ Our monitoring strategy is according to these recommendations. In 2016, European Heart Rhythm Association and European Stroke Organisation recommended screening for $\mathrm{AF}$ in patients who had an ischaemic stroke or TIA by short-term ECG recording followed by continued ECG monitoring for at least 72 hours. ${ }^{7}$ Several studies published within recent years encourage longer monitoring time of patients who had a cryptogenic stroke since it reveals significantly more AF events. The American Heart Association recommends 30-day monitoring of patients who had a stroke since $2014 .^{8}$

It is not straightforward to offer 30-day continuous ECG recording for all patients who had a stroke. This would necessitate major resources for manual analysis of recordings. Most continuous ECG recording devices in use are not capable of such long-time recording. Incidence of skin reactions from wearing continuous ECG recording electrodes is not negligible. In 2014, Tu et $a l^{21}$ reported that 28-day continuous ECG recording was poorly tolerated in patients who had a cryptogenic stroke, with only $40 \%$ completing the recording period; remaining patients were excluded mainly due to skin irritation and also artefacts caused by loose electrodes and trouble sleeping with the device caused exclusions. Higher patient comfort and compliance was observed with a specially designed textile wearable continuous ECG recorder, resulting in $84 \%$ of patients who had a cryptogenic stroke completing 28 -day recording. ${ }^{22}$ Gain of prolonging 

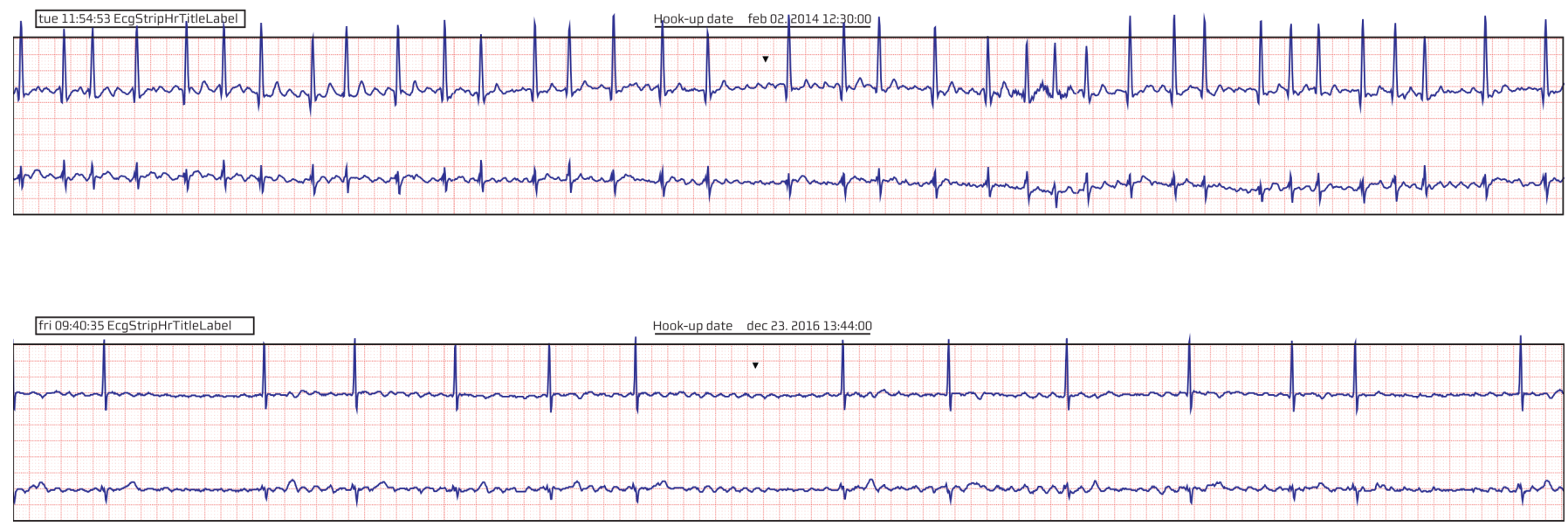

Figure 4 (A and B) Two of six patients diagnosed with AF on 48-hour continuous ECG recording but missed by ELR. AF, atrial fibrillation; ELR, external loop recorder.

continuous ECG recording in AF detection is currently explored in the ongoing German Impact of standardized MONitoring for Detection of Atrial Fibrillation in Ischemic Stroke trial. ${ }^{23}$

Considering American guidelines, automatically detecting ELR devices are attractive alternatives to continuous ECG recording, being able to record for 30 days, associated with less patient discomfort and less costly to analyse. However, the type of ELR used must identify AF reliably. For longer term monitoring, implantable loop recorders are superior with respect to patient comfort and reliability ${ }^{24}$ but more expensive and requiring surgery. Documenting cost-effectiveness of ILR in patients who had a stroke is still needed. Ambulatory telemetry has been tested only in small cohorts ${ }^{25}$ and requires personnel validating the results manually. Prolonged inpatient telemetry has been studied and results in higher rates of AF detection in patients who had a stroke. ${ }^{26}$ However, in Denmark, the average patient who had a stroke is admitted to hospital for 2 days and patients who had a TIA even shorter. A wealth of different devices recording heart rhythm is being marketed: mobile phones, thumb-ECG, earlobe sensors, smartwatches and automated blood pressure monitors. For such devices, performance should be tested before using for screening patients who had a stroke.

\section{Study strengths}

The essential strength of this study is simultaneous ELR monitoring and continuous ECG recording in patients who had an acute stroke and TIA, permitting true validation of ELR against the gold standard of continuous ECG recording. A large number of patients were included, allowing for more reliable statistical estimates. Investigators analysing continuous ECG recordings were blinded to ELR tracing results and vice versa. The study was performed in Denmark where the healthcare system is free of charge. All Danish patients who had a stroke and TIA are referred to neurological departments and treated according to national and European guidelines without regard to social and economic status. Therefore, the patients represent an unselected stroke population and the results are considered generalisable.

\section{Study limitations}

Not all patients consented, and few patients were not asked because low compliance was anticipated. In periods of high occupancy, we had shortage of continuous ECG recorders and not all patients could be included. It is, however, not likely that inclusion of these patients would have changed the comparison between ELR and continuous ECG recordings. We excluded 95 patients with ELR started incorrectly, resulting in empty recordings. As ELR recordings are not real-time transmissions, quality of recording could not be controlled until after detachment of device. An ELR recorder easier to use might have reduced this problem. Planning the study, we expected AF prevalence of $10 \%$ in 48 -hour continuous ECG, but we observed a prevalence of $2.7 \%$. This discrepancy should be taken into account interpreting positive predictive value and negative predictive value reported, as accuracy of both decreases with more rare observations. Furthermore, we did not calculate our sample size for specificity.

The ELR tested in this study used only single-lead recording from two electrodes. Using another type of ELR recording with more than one lead may have allowed better differentiation between $\mathrm{AF}$ and non-AF rhythms. We used the generally accepted definition of AF at $30 \mathrm{~s}$ duration. It cannot be ruled out that the ELR may have performed better with respect to properly detecting longer AF episodes.

\section{SUMMARY}

Automatic AF detection with ELR results in an AF diagnosis in more than five patients without AF for each patient with AF as verified in continuous ECG. For adjudicated AF detection by ELR, sensitivity was confirmed to $85 \%$ and specificity $98 \%$. Automatic ELR as investigated in this study may be considered to rule out AF, but it is not suitable as a single monitoring device for AF screening in patients early after stroke.

Therefore, this type of automatic ELR, however, is not suitable.

The author group has no economic interest in the research results and received no financial support from ECG recorder or ELR provider. The corresponding author has the right to grant on behalf of all authors and does grant on behalf of all authors an exclusive licence on a worldwide basis to the BMJ Publishing Group Ltd (BMJPGL) and its licencees to permit this article to be published in Heart and any other BMJPGL products to exploit all subsidiary rights. 


\section{Key messages}

\section{What is already known about this subject?}

- It is well documented that oral anticoagulation treatment reduces stroke risk significantly in patients with atrial fibrillation (AF) and detection of AF in patients who had a stroke is of utmost importance.

- There is, however, no consensus about best method to detect non-persistent AF.

\section{What might this study add?}

- Automatic AF detection with external loop recorder (ELR) results in an AF diagnosis in more than five patients without AF for each patient with AF as verified in continuous ECG. For adjudicated AF detection by ELR, sensitivity was $84 \%$, positive predictive value was $56 \%$ and specificity was $98 \%$.

\section{How might this impact on clinical practice?}

- Automatic ELR as investigated in this study may be considered to rule out $A F$, but it is not suitable as a single monitoring device for AF screening in patients early after stroke.

Acknowledgements We would like to thank nurses at our Department of Neurology, especially Ida Tølbøl, Anette Smidth, Birgitte Brøgger, Mette Jensen; nurses at Department of Cardiology: Marie Jensen, Britta Graversen, Helle Schlosser, Ruth Larsen, Birgit Pedersen and Mette Mark; and Mads Liseborg on graphics.

Contributors MHS contributed in literature search, fund applications and obtaining permits, figures, study design, data collection, data analysis, data interpretation and writing. JCN contributed in literature search, figures, study design, data analysis, data interpretation, writing and guidance on cardiological aspects. OM contributed in figures, study design, data interpretation, writing and guidance on cardiological aspects. DD contributed in study design, writing and guidance on neurologic aspects. BFS contributed in data collection, writing and guidance on neurologic aspects.

Funding This work was supported by Health Research Fund of Central Denmark Region (1-31-72-15-14), Danish Heart Foundation (14-R97-A5075-22884/17R115-A7606-22069) and Aase and Ejnar Danielsen Foundation (10-001847). Novo Nordisk Foundation (NNF160C0018658) and an institutional unrestricted grant from Abbott, Denmark, supported JCN.

Competing interests None declared.

Patient consent for publication Not required.

Provenance and peer review Not commissioned; externally peer reviewed.

\section{REFERENCES}

1 Khoo CW, Lip GY. Clinical outcomes of acute stroke patients with atrial fibrillation. Expert Rev Cardiovasc Ther 2009;7:371-4.

2 Marini C, De Santis F, Sacco S, et al. Contribution of atrial fibrillation to incidence and outcome of ischemic stroke: results from a population-based study. Stroke 2005;36:1115-9.

3 January CT, Wann LS, Alpert JS, et al. 2014 AHA/ACC/HRS guideline for the management of patients with atrial fibrillation: a report of the American College of Cardiology/American heart association task force on practice guidelines and the heart rhythm society. J Am Coll Cardiol 2014;64:e1-76.

4 Sposato LA, Cipriano LE, Saposnik G, et al. Diagnosis of atrial fibrillation after stroke and transient ischaemic attack: a systematic review and meta-analysis. Lancet Neurol 2015;14:377-87.
5 Thygesen SK, Frost L, Eagle KA, et al. Atrial fibrillation in patients with ischemic stroke: a population-based study. Clin Epidemiol 2009;1:55-65.

6 EAFT (European Atrial Fibrillation Trial) Study Group. Secondary prevention in nonrheumatic atrial fibrillation after transient ischaemic attack or minor stroke. Lancet 1993;342:1255-62.

7 Mairesse GH, Moran P, Van Gelder IC, et al. Screening for atrial fibrillation: a European Heart Rhythm Association (EHRA) consensus document endorsed by the Heart Rhythm Society (HRS), Asia Pacific Heart Rhythm Society (APHRS), and Sociedad Latinoamericana de Estimulación Cardíaca y Electrofisiología (SOLAECE). Europace 2017;19:1589-623.

8 Kernan WN, Ovbiagele B, Black HR, et al. Guidelines for the prevention of stroke in patients with stroke and transient ischemic attack: a guideline for healthcare professionals from the American heart association/American stroke association. Stroke 2014;45:2160-236.

9 Rankin AJ, Tran RT, Abdul-Rahim AH, et al. Clinically important atrial arrhythmia and stroke risk: a UK-wide online survey among stroke physicians and cardiologists. QJM 2014; 107:895-902.

10 Roten L, Schilling M, Häberlin A, et al. Is 7-day event triggered ECG recording equivalent to 7-day Holter ECG recording for atrial fibrillation screening? Heart 2012;98:645-9.

11 Bell C, Kapral M. Use of ambulatory electrocardiography for the detection of paroxysmal atrial fibrillation in patients with stroke. The Canadian Journal of Neurological Sciences 2000;27:25-31.

12 Sejr MH, Nielsen JC, Damgaard D, et al. Atrial fibrillation detected by external loop recording for seven days or two-day simultaneous Holter recording: A comparison in patients with ischemic stroke or transient ischemic attack. J Electrocardiol 2017; 50:287-93.

13 Müller A, Scharner W, Borchardt T, et al. Reliability of an external loop recorder for automatic recognition and transtelephonic ECG transmission of atrial fibrillation. J Telemed Telecare 2009;15:391-6.

14 Takagi T, Miyazaki S, Kusa S, et al. Role of extended external auto-triggered loop recorder monitoring for atrial fibrillation. Circ J 2014;78:2637-42.

15 Chow GV, Marine JE, Fleg JL. Epidemiology of arrhythmias and conduction disorders in older adults. Clin Geriatr Med 2012;28:539-53.

16 Bybee KA, Prasad A. Stress-related cardiomyopathy syndromes. Circulation 2008;118:397-409.

17 Diener HC, Eikelboom J, Connolly SJ, et al. Apixaban versus aspirin in patients with atrial fibrillation and previous stroke or transient ischaemic attack: a predefined subgroup analysis from AVERROES, a randomised trial. Lancet Neurol 2012;11:225-31.

18 Diener HC, Connolly SJ, Ezekowitz MD, et al. Dabigatran compared with warfarin in patients with atrial fibrillation and previous transient ischaemic attack or stroke: a subgroup analysis of the RE-LY trial. Lancet Neurol 2010;9:1157-63.

19 Hart RG, Sharma M, Mundl H, et al. Rivaroxaban for stroke prevention after embolic stroke of undetermined source. N Engl J Med 2018.

20 European Stroke Organisation (ESO) Executive Committee ESO Writing Committee. Guidelines for management of ischaemic stroke and transient ischaemic attack 2008. Cerebrovasc Dis 2008;25:457-507.

21 Tu HT, Spence S, Kalman JM, et al. Twenty-eight day holter monitoring is poorly tolerated and insensitive for paroxysmal atrial fibrillation detection in cryptogenic stroke. Intern Med J 2014;44:505-8.

22 Pagola J, Juega J, Francisco-Pascual J, et al. Yield of atrial fibrillation detection with textile wearable holter from the acute phase of stroke: pilot study of crypto-AF registry. Int J Cardiol 2018;251:45-50.

23 Haeusler KG, Kirchhof P, Heuschmann PU, et al. Impact of standardized MONitoring for Detection of Atrial Fibrillation in Ischemic Stroke (MonDAFIS): Rationale and design of a prospective randomized multicenter study. Am Heart J 2016;172:19-25.

24 Hindricks G, Pokushalov E, Urban L, et al. Performance of a new leadless implantable cardiac monitor in detecting and quantifying atrial fibrillation: results of the XPECT trial. Circ Arrhythm Electrophysiol 2010;3:141-7.

25 Kamel H, Smith WS. Detection of atrial fibrillation and secondary stroke prevention using telemetry and ambulatory cardiac monitoring. Curr Atheroscler Rep 2011;13:338-43

26 Sposato LA, Klein FR, Jáuregui A, et al. Newly diagnosed atrial fibrillation after acute ischemic stroke and transient ischemic attack: importance of immediate and prolonged continuous cardiac monitoring. J Stroke Cerebrovasc Dis 2012;21:210-6. 